\title{
Fungal DNA, allergens, mycotoxins and associations with asthmatic symptoms among pupils in schools from Johor Bahru, Malaysia.
}

\begin{abstract}
While there is a large variation of prevalence of asthma symptoms worldwide, what we do know is that it is on the rise in developing countries. However, there are few studies on allergens, moulds and mycotoxin exposure in schools in tropical countries. The aims were to measure selected fungal DNA, furry pet allergens and mycotoxins in dust samples from schools in Malaysia and to study associations with pupils' respiratory health effects. Eight secondary schools and 32 classrooms in Johor Bahru, Malaysia were randomly selected. A questionnaire with standardized questions was used for health assessment in 15 randomly selected pupils from each class. The school buildings were inspected and both indoor and outdoor climate were measured. Dust samples were collected by cotton swabs and Petri dishes for fungal DNA, mycotoxins and allergens analysis. The participation rate was $96 \%$ (462/480 invited pupils), with a mean age of 14yr (range 14-16). The pupils mostly reported daytime breathlessness (41\%), parental asthma or allergy (22\%), pollen or pet allergy $(21 \%)$ and doctor-diagnosed asthma (13\%) but rarely reported night-time breathlessness $(7 \%)$, asthma in the last 12 months $(3 \%)$, medication for asthma (4\%) or smoking (5\%). The inspection showed that no school had any mechanical ventilation system, but all classrooms had openable windows that were kept open during lectures. The mean building age was 16yr (range 3-40) and the mean indoor and outdoor CO2 levels were 492ppm and 408ppm, respectively. The mean values of indoor and outdoor temperature and relative humidity were the same, $29^{\circ} \mathrm{C}$ and $70 \%$ respectively. In cotton swab dust samples, the Geometric Mean (GM) value for total fungal DNA and Aspergillus/Penicillium (Asp/Pen) DNA in swab samples (Cell Equivalents $(\mathrm{CE}) / \mathrm{m} 2$ ) was $5.7 * 108$ and $0.5 * 108$, respectively. The arithmetic mean $(\mathrm{CE} / \mathrm{m} 2)$ for Aspergillus versicolor DNA was 8780 , Stachybotrys chartarum DNA was 26 and Streptomyces DNA was 893. The arithmetic means $(\mathrm{pg} / \mathrm{m} 2)$ for the mycotoxins sterigmatocystin and verrucarol were 2547 and 17, respectively. In Petri dish dust samples, the GM value for total fungal DNA and Asp/Pen DNA (CE/m2 per day) was $9.2 * 106$ and $1.6 * 106$, respectively. The arithmetic mean $(\mathrm{CE} / \mathrm{m} 2$ per day) for A. versicolor DNA was 1478 , S. chartarum DNA was 105 and Streptomyces DNA was 1271, respectively. The GM value for cat(Fel d1) allergen was $5.9 \mathrm{ng} / \mathrm{m} 2$ per day. There were positive associations between A. versicolor DNA, wheeze and daytime breathlessness and between Streptomyces DNA and doctor-diagnosed asthma. However, the associations were inverse between S. chartarum DNA and daytime breathlessness and between verrucarol and daytime breathlessness. In conclusion, fungal DNA and cat allergen contamination were common in schools from Malaysia and there was a high prevalence of respiratory symptoms among pupils. Moreover, there were associations between levels of some fungal DNA and reported respiratory health in the pupils.
\end{abstract}

Keyword: Allergens; Asthma; Bacteria; Fungal DNA; Indoor environments; Mycotoxins; Quantitative PCR; Respiratory symptoms; School environments. 